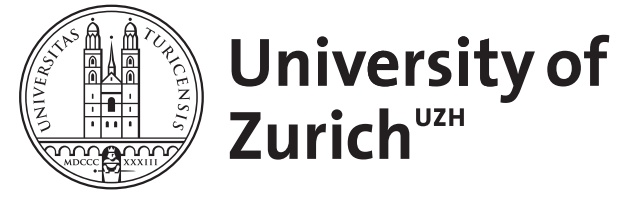
Archive

University of Zurich

University Library

Strickhofstrasse 39

CH-8057 Zurich

www.zora.uzh.ch

Year: 2016

\title{
Strafrecht am Ende des Lebens - Sterbehilfe und Hilfe zum Suizid in der Schweiz
}

Tag, Brigitte

DOI: https://doi.org/10.1515/zstw-2016-0004

Posted at the Zurich Open Repository and Archive, University of Zurich ZORA URL: https://doi.org/10.5167/uzh-171920

Journal Article

Published Version

Originally published at:

Tag, Brigitte (2016). Strafrecht am Ende des Lebens - Sterbehilfe und Hilfe zum Suizid in der Schweiz. Zeitschrift für die gesamte Strafrechtswissenschaft, 128(1):73-88.

DOI: https://doi.org/10.1515/zstw-2016-0004 
Professor Dr. iur. utr. Brigitte Tag*

\section{Strafrecht am Ende des Lebens - Sterbehilfe und Hilfe zum Suizid in der Schweiz}

\section{Ausgangslage}

Die rechtliche und ethische Einordnung der Sterbehilfe und der Hilfe zum Suizid in der Schweiz ist wie in vielen anderen Ländern komplex. Regelungen der Bundesverfassung, der Konvention zum Schutze der Menschenrechte und Grundfreiheiten, des Straf-, Zivil- und des Öffentlichen Rechts auf Bundesebene, aber auch kantonale Bestimmungen und ethische Normen ergeben ein facettenreiches Regelwerk. In seinem Mittelpunkt stehen freilich einige zentrale Fragen, deren Antworten weitreichende Bedeutung entfalten. Es geht hierbei um das Recht auf Leben und die persönliche Freiheit, einschließlich der Autonomie, die Menschenwürde, aber auch die Glaubens- und Gewissensfreiheit, den Schutz der Gesundheit und die Hilfe in Notlagen. Diese Grundrechte bzw. Sozialziele finden ihre Konkretisierungen in den Normen des Bundes wie dem Strafgesetzbuch, dem Zivilgesetzbuch, dem Krankenversicherungsrecht und den kantonalen Regelungen. Der Bereich des Gesundheitsrechts liegt primär in der Zuständigkeit der Kantone, soweit der Bund keine expliziten Regelungen hierzu erlassen hat, Art. $42 \mathrm{ff} . \mathrm{BV}^{1}$.

Das in der generellen Sterbehilfediskussion prominent erörterte Recht, die Umstände des eigenen Todes („right to die“) selbst zu bestimmen, kennt die Schweizer Rechtsordnung im Sinne eines selbstständigen Grundrechts nicht. Eine solche Gewährleistung wird jedoch aus der Zusammenschau etlicher verfassungsrechtlicher Bestimmungen abgeleitet, wobei die hierbei gesetzten immanenten und ausdrücklichen Grenzen zu beachten sind. Maßgebend sind namentlich der Schutz der Menschenwürde (Art. 7 BV ) und des Selbstbestimmungsrechts, wel-

1 Bundesverfassung der Schweizerischen Eidgenossenschaft vom 18. 04. 1999, SR 101.

2 Art. 7 BV „Menschenwürde“ lautet wie folgt: „Die Würde des Menschen ist zu achten und zu schützen“.

*Kontaktperson: Brigitte Tag, Inhaberin des Lehrstuhls für Strafrecht, Strafprozessrecht und Medizinrecht an der Universität Zürich 
ches sich aus dem Recht der persönlichen Freiheit (Art. 10 Abs. 2 BV³) ableitet. Nach der Rechtsprechung des Bundesgerichts garantiert die persönliche Freiheit alle Aspekte, die bestimmend für die Entfaltung der Persönlichkeit sind ${ }^{4}$. Sie gewährleistet zudem ein Mindestmaß an persönlicher Entfaltungsmöglichkeit und die Achtung der hiermit in der Regel verbundenen Fähigkeit, tatsächliche Umstände zu würdigen und danach zu handeln. Aber auch das Recht auf Glaubens- und Gewissensfreiheit (Art. $15 \mathrm{BV}^{5}$ ) entfaltet bezüglich der Bestimmung der Umstände des eigenen Todes Relevanz $z^{6}$. Neben den verfassungsimmanenten Schranken regelt Art. $36 \mathrm{BV}^{7}$ die Voraussetzungen allfälliger Grundrechtseinschränkungen. Die Grenze der Zulässigkeit von Eingriffen in den Schutzbereich ist im Einzelfall und in Bezug auf die Art und Intensität der Beeinträchtigung sowie im Hinblick auf eine allfällige besondere Schutzwürdigkeit der betroffenen Person zu konkretisieren ${ }^{8}$.

Ergänzt werden die nationalen Regelungen durch Bestimmungen des internationalen Rechts, völkerrechtliche Verträge sowie die Menschenrechtsgarantien

3 Art. 10 Abs. 2 BV „Recht auf Leben und auf persönliche Freiheit“ lautet wie folgt: „Jeder Mensch hat das Recht auf persönliche Freiheit, insbesondere auf körperliche und geistige Unversehrtheit und auf Bewegungsfreiheit“".

4 Vgl. z.B. BGE 133 I 58, 66 m.w. N., bezogen auf Art. 10 Abs. 2 BV.

5 Art. 15 BV „Glaubens- und Gewissensfreiheit“ lautet wie folgt:

Abs. 1 „Die Glaubens- und Gewissensfreiheit ist gewährleistet“.

Abs. 2 ,Jede Person hat das Recht, ihre Religion und ihre weltanschauliche Überzeugung frei zu wählen und allein oder in Gemeinschaft mit anderen zu bekennen“.

Abs. 3 „Jede Person hat das Recht, einer Religionsgemeinschaft beizutreten oder anzugehören und religiösem Unterricht zu folgen“.

Abs. 4 „Niemand darf gezwungen werden, einer Religionsgemeinschaft beizutreten oder anzugehören, eine religiöse Handlung vorzunehmen oder religiösem Unterricht zu folgen“.

6 Zum Umgang mit der Leiche vgl. z.B. Tag, in: Tag/Mausbach/Moch (Hrsg.), Autopsie und Religion, 2013, S. 37; zum Hungerstreik im Gefängnis vgl. Tag, in: Tag/Gross (Hrsg.), Tod im Gefängnis, 2012, S. 23. Hierbei ist freilich zu beachten, dass das Bundesgericht dem Inhaftierten letztlich das Recht, sich im Strafvollzug zu Tode zu hungern, abgesprochen hat. Vgl. zum sehr umstrittenen BG-Entscheid vom 16. 11. 2010 im Fall „Rappaz“ mit krit. Besprechung Tag, forumpoenale 3 (2011), S. 153.

7 Art. 36 BV „Einschränkungen von Grundrechten“ lautet wie folgt:

Abs. 1 „Einschränkungen von Grundrechten bedürfen einer gesetzlichen Grundlage. Schwerwiegende Einschränkungen müssen im Gesetz selbst vorgesehen sein. Ausgenommen sind Fälle ernster, unmittelbarer und nicht anders abwendbarer Gefahr“"

Abs. 2 „Einschränkungen von Grundrechten müssen durch ein öffentliches Interesse oder durch den Schutz von Grundrechten Dritter gerechtfertigt sein“.

Abs. 3 „Einschränkungen von Grundrechten müssen verhältnismässig sein“.

Abs. 4 „Der Kerngehalt der Grundrechte ist unantastbar“.

8 Vgl. z.B. BGE 133 I 58, 66 m. zahlr. N. 
der EMRK. Die EMRK ist für die Schweiz seit dem 28. November 1974 in $\mathrm{Kraft}^{9}$ und gewährleistet in Art. 8 Ziff. 1 EMRK das Recht auf Achtung des Privatlebens. Dieser Schutzbereich, er überschneidet sich mit demjenigen von Art. 10 Abs. 2 BV, umfasst auch das Recht des Patienten über die Art und den Zeitpunkt der Beendigung des eigenen Lebens selbst entscheiden $\mathrm{zu}$ können ${ }^{10}$. Die Schweiz wurde vom EGMR bislang aber nicht rechtskräftig verpflichtet, ein eigenes Gesetz zur Regelung allfälliger Rahmenbedingungen für den Bereich der Sterbehilfe auszuarbeiten $^{11}$.

Diese Garantien sind bislang als Abwehrrechte zu verstehen. Würde es einen Anspruch, d.h. ein positives Recht, darauf geben, dass der Staat Bedingungen schaffen müsste, unter denen der Suizid besonders leicht $\mathrm{zu}$ begehen ist, indem z. B. nichtärztliche Personen einen bedingungslosen Anspruch auf das bei der Sterbehilfe eingesetzte Natrium-Pentobarbital haben und durchsetzen könnten, würde neben anderen Implikationen das Risiko geschaffen werden, jemanden auch gegen den eigenen Willen zur Mitwirkung am Suizid zu verpflichten. Ein solches Anspruchsrecht wird in der Schweiz jedoch nicht anerkannt. Es besteht demzufolge auch kein Anspruch der sterbewilligen Person auf Hilfe durch den Staat oder eine Organisation, sofern sie selbst nicht (mehr) in der Lage ist, ihrem Leben ein Ende zu setzen ${ }^{12}$.

\section{Sterbehilfe allgemein, Fremdtötung und Teilnahme an der Selbsttötung}

Die Sterbehilfedebatte in der Schweiz kann in zwei große Themenkreise aufgeteilt werden. Es geht zum einen um den Bereich der Fremdtötung, zum anderen um die Selbsttötung und einer allfällig dazu geleisteten Unterstützung. Von Seiten des schweizerischen Strafgesetzgebers besteht ein eindeutiges Verbot der aktiven Tötung und damit auch der direkten aktiven Sterbehilfe im Sinne der Fremdtötung. Dieses Verbot betrifft das Handeln als Täter und richtet sich an jedermann. Vom Verbot betroffen sind auch die Familienangehörigen, Freunde, Sterbehelfer, der behandelnde Arzt ${ }^{13}$ oder das Pflegepersonal.

9 Konvention zum Schutze der Menschenrechte und Grundfreiheiten vom 28. 11. 1974, SR 0.101.

10 Vgl. z. B. EGMR, Application no. 2346/02, 29. 07. 2002 - Pretty/United Kingdom.

11 EGMR, Application no. 67810/10, 30. 09. 2014 - Gross/Switzerland.

12 BGE 133 I 58, 67-68.

13 So Art. 17 Abs. 2 der Standesordnung der Schweizer Ärztinnen und Ärzte, FMH. 


\section{Abgrenzung von Fremdtötung und Suizidbeteiligung}

\section{a) Selbsttötung und Suizidbeteiligung}

Die Abgrenzung von Fremdtötung und Suizidbeteiligung erfolgt in der Schweiz nach den allgemeinen Bestimmungen des Strafrechts, namentlich den Regeln der Täterschaft und Teilnahme ${ }^{14}$. Hat die Person, die beabsichtigt, Suizid zu begehen, Tatherrschaft und damit Tatmacht über dieses Geschehen, liegt eine eigenverantwortliche Selbsttötung vor, falls der Tatentschluss auch umgesetzt wird. Voraussetzung ist insbesondere die Urteilsfähigkeit der betreffenden Person, das Fehlen von auf das Suizidgeschehen bezogenen Willensmängeln sowie das „in den Händen Halten“ des Geschehens bis zum „Point of no return“. Die freiverantwortliche Selbsttötung ist in der Schweiz nicht pönalisiert. Die akzessorische Teilnahme hierbei ist nur ${ }^{15}$ strafbar, wenn die Voraussetzungen von Art. $115 \mathrm{StGB}^{16}$, Verleitung und Beihilfe zum Selbstmord aus selbstsüchtigen Beweggründen ${ }^{17}$, vorliegen. Dieser Tatbestand wurde geschaffen, um dem hohen Rang des Rechtsguts Leben sowie einer allfälligen Missbrauchsgefahr der Teilnahme zur Selbsttötung entgegenzutreten. Als einschränkendes Merkmal ist gefordert, dass die Beteiligung aus selbstsüchtigen Motiven erfolgt. Die Rechtsprechung definiert den Bereich der selbstsüchtigen Beweggründe „wenn der Täter einen persönlichen Vorteil (materieller oder ideeller Natur) verfolgt" ${ }^{\text {“18. Die }}$ Gesetzesmaterialien nennen als Beispiel die Unterstützung einer Person beim Selbstmord, die der Täter zu beerben hofft ${ }^{19}$. Die Verleitung im Sinne der Anstiftung $^{20}$ zum Selbstmord muss das Hervorrufen eines Selbsttötungsentschlus-

14 Donatsch/Tag, Strafrecht I, 9. Aufl. 2013, § 14ff.; BGE 133 I 58, 72; BGE 129 IV 124, 126; BGer vom 25. 02. 2005, 6P124/2004.

15 Die Botschaft des Bundesrates an die Bundesversammlung zu einem Gesetzesentwurf enthaltend das schweizerische Strafgesetzbuch vom 23. 07. 1918, S. 32 (BBl 1918 IV 1), unterstreicht, dass die Teilnahme an der Selbsttötung eine „Freundestat“ sein könne, mithin ein tatbestandsausschließender positiver Zweck der Tat vorliege, der einer Bestrafung des Täters zuwiderlaufen würde. Auf eine generelle Strafbarkeit der Teilnahme am Suizid wurde daher verzichtet.

16 Art. 115 StGB lautet wie folgt: „Wer aus selbstsüchtigen Beweggründen jemanden zum Selbstmord verleitet oder ihm dazu Hilfe leistet, wird, wenn der Selbstmord ausgeführt oder versucht wurde, mit Freiheitsstrafe bis zu fünf Jahren oder Geldstrafe bestraft“.

17 Tag, in: Kuhn/Poledna (Hrsg.), Arztrecht in der Praxis, 2007, S. 742.

18 Vgl. BGer vom 17. 11. 2011, 1B_516/2011, E. 2.4; Schwarzenegger, in: Basler Kommentar Strafrecht II, 3. Aufl. 2013, Art. 115 Rdn. 14.

19 Botschaft (Anm. 15), S. 32; Tag, in: Wehrli/Sutter/Kaufmann (Hrsg.), Der organisierte Tod, Sterbehilfe und Selbstbestimmung am Lebensende - Pro und Contra, 2015, S. $47 \mathrm{ff}$.

20 Vgl. Art. 24 StGB. 
ses bei einem anderen Menschen und dessen tatherrschaftlich und eigenverantwortlich ausgeführten Suizid bewirken. Ausschlaggebend ist, dass die angestiftete Person zumindest den letzten Schritt zur Selbsttötung alleine ausführt und diesen auch eigenverantwortlich abbrechen kann ${ }^{21}$. Auch die Variante Beihilfe ${ }^{22}$ zum Selbstmord bedingt, dass zumindest der letzte Schritt der Selbsttötung durch die sterbewillige Person tatherrschaftlich und eigenverantwortlich durchgeführt wird. Der Gehilfe leistet dabei einen zumindest fördernden Beitrag, welcher auch in einer psychischen Unterstützung bestehen kann ${ }^{23}$. Liegen keine selbstsüchtigen Beweggründe vor und wird einer sterbewilligen Person z. B. ein tödliches Mittel verschafft bzw. werden ihr die nötigen Hinweise zur Durchführung des Suizids gegeben, ist diese Art der Unterstützung straflos.

Auch ein Arzt bzw. eine Ärztin kann nach dem Schweizer Recht Suizidbeihilfe leisten. Das Strafgesetzbuch enthält hierzu keine Einschränkungen. Das Standesrecht der Ärzte unterstellt eine allfällige Hilfeleistung bzw. die Verweigerung einer solchen Unterstützung dem Gewissensentscheid. Zwar wird klar darauf hingewiesen, dass die Rolle des Arztes bei Patienten am Lebensende sich auf die Linderung von Symptomen und die Begleitung von Patienten bezieht und nicht darauf, von sich aus Suizidbeihilfe anzubieten. Bei dauerhaftem und ernsthaftem Wunsch des Patienten am Lebensende wird die persönliche Gewissensentscheidung des Arztes, im Einzelfall Beihilfe zum Suizid zu leisten, respektiert. Entschließt sich ein Arzt zur Suizidbeihilfe, gelten folgende Voraussetzungen: Die Erkrankung des Patienten rechtfertigt die Annahme, dass das Lebensende nahe ist. Alternative Möglichkeiten der Hilfestellung wurden erörtert und soweit gewünscht auch eingesetzt. Der Patient ist urteilsfähig, sein Wunsch wohlerwogen und ohne äußeren Druck entstanden sowie dauerhaft. Die Überprüfung erfolgt durch eine unabhängige Drittperson, welche nicht zwingend ein Arzt sein muss $^{24}$. Sind diese Bedingungen eingehalten, ist das Verhalten des Arztes nicht standeswidrig.

Art. 115 StGB ist als abschließend zu bewerten ${ }^{25}$. Wollte man daneben auch die Straftatbestände, wie z.B. die vorsätzliche Tötung durch Unterlassen, Art. 111 ff. i. V.m. Art. 11 StGB, anwenden, würde der nicht gegen den beabsichtigten oder eingeleiteten Selbstmord einschreitende Garant einer strengeren straf-

21 Donatsch, Strafrecht III, 10. Aufl. 2013, § 1, 6.1.

22 Rechtsgedanke Art. 25 StGB.

23 Schwarzenegger, in: BSK (Anm. 18), Art. 115 Rdn. 9-10.

24 Näher Art. 18 Standesordnung der FMH i. V.m. der SAMW-Richtlinie Betreuung von Patientinnen und Patienten am Lebensende, Kommentar 4.1.

25 I. d. S. auch Stratenwerth/Jenny/Bommer, Schweizerisches Strafrecht, Besonderer Teil I, 7. Aufl. 2010, § 1 Rdn. 53, $62 \mathrm{f}$. 
rechtlichen Verantwortlichkeit unterstehen als derjenige, welcher dem Suizidalen dafür Mittel zur Verfügung stellt ${ }^{26}$. Der aus selbstlosen Gründen Hilfe leistende Garant wäre nach Art. 115 StGB straffrei, nach Art. $111 \mathrm{ff}$. StGB, insbesondere Art. 113 StGB, Art. 11 StGB, hingegen wegen Unterlassens allfälliger Hilfe strafbar. Ein solches Ergebnis wäre widersprüchlich und ist sowohl aus systematischen wie teleologischen Gründen abzulehnen.

Die straflose Teilnahme zum Suizid bedingt, dass der Suizid eigenverantwortlich und tatherrschaftlich durchgeführt wird. Ist der Suizident in Bezug auf seine Selbsttötungshandlung urteilsunfähig, weil er nicht in der Lage ist, die Bedeutung und Tragweite der Selbsttötungshandlung und des zum Tode führenden Geschehensablaufs zu verstehen oder nach dieser Einsicht $\mathrm{zu}$ handeln ${ }^{27}$, kommt allenfalls mittelbare Täterschaft der unterstützenden Person in Betracht, sofern der Suizident als willenloses oder zumindest nicht vorsätzlich handelndes Werkzeug benutzt wird, um sich selbst $z u$ töten ${ }^{28}$. Unterliegt die unterstützende Person einem bei pflichtgemäßer Vorsicht vermeidbarem Irrtum über die Freiverantwortlichkeit des Suizidenten, ist eine fahrlässige Tötung in Betracht zu ziehen, Art. 13, Art. 117 StGB. Aufgrund der Relativität der Urteilsfähigkeit ist diese immer im Hinblick auf die konkrete Handlung und die jeweilige Situation, in welcher sich die Person befindet, zu beurteilen. Insbesondere bei psychischen Erkrankungen und depressiven Zuständen muss die Urteilsfähigkeit der suizidwilligen Person mit besonderer Umsicht abgeklärt werden. Das Bundesgericht anerkennt, dass auch im Falle einer unheilbaren, dauerhaften, schweren psychischen Störung ein strafloser assistierter Suizid möglich sein soll. Es mahnt jedoch zur äußersten Zurückhaltung und fordert eine genaue Abklärung, ob der Sterbewunsch therapierbar ist oder auf einer wohlerwogenen und dauerhaften Entscheidung beruhe, welcher $\mathrm{zu}$ respektieren sei. Um dies beurteilen zu können, sei ein vertieftes psychiatrisches Fachgutachten notwen$\operatorname{dig}^{29}$.

Finden nicht eingeweihte Dritte eine Person auf, die infolge eines Suizidversuches in unmittelbarer Lebensgefahr schwebt, muss Hilfe geleistet werden. Denn bestehen keine verlässlichen und belastbaren Anhaltspunkte dafür, dass ein freiverantwortlicher Suizid vorliegt, besteht zumindest ${ }^{30}$ die allgemeine Unter-

26 Stratenwerth/Jenny/Bommer, Bes. Teil (Anm. 25), §1 Rdn. 53; ebenso Donatsch, Sorgfaltsbemessung und Erfolg beim Fahrlässigkeitsdelikt, 1987, S. 350 f., beide m.w. N.

27 BGer vom 11. 6. 2009, 6B_48/2009, E. 2.

28 Donatsch/Tag, Strafrecht I (Anm. 14), § 15, 3.1.

29 BGE 133 I 58, $74 \mathrm{ff}$.

30 Bei Garanten kommt eine Strafbarkeit aus dem unechten Unterlassungsdelikt, d.h. Art. 111, Art. 11 StGB, in Betracht. 
stützungspflicht nach Art. $128 \mathrm{StGB}$, Unterlassung der Nothilfe ${ }^{31}$, selbst wenn sich im Nachhinein herausstellt, dass die Situation eine andere war. Schwierig gestaltet sich die Situation für Rettungssanitäter ${ }^{32}$ und Ärzte, die in einer Akutsituation gerufen werden. Lässt sich hier in der Kürze der zur Verfügung stehenden Eingriffszeit nicht zuverlässig abklären, ob eine gültige Patientenverfügung ein Eingreifen untersagt, sind sie als Garant für das Leben des Hilfesuchenden zum Handeln verpflichtet, d.h. grundsätzlich muss ein Reanimationsversuch durchgeführt werden ${ }^{33}$. Ein Stempel auf der Brust des Hilfsbedürftigen mit der Aufschrift „No CPR“ (Cardio-Pulmonary Resuscitation, Herz-Lungen-Wiederbelebung) kann aufgrund des Missbrauchsrisikos und der fehlenden, aber gemäß Art. 371 ZGB konstituierenden Voraussetzungen einer Patientenverfügung, welche schriftlich zu verfassen, zu datieren und handschriftlich zu unterzeichnen ist, diese Aufschrift zwar ergänzen, aber nicht ersetzen ${ }^{34}$.

\section{b) Sterbehilfeorganisationen}

Am Maßstab von Art. 115 StGB ist auch das Handeln von Sterbehilfeorganisationen zu messen ${ }^{35}$. Da ihr Tätigwerden in der Regel nicht kostenfrei ist, hat sich die Frage daran entzündet, ob das erhobene Entgelt, das sich je nach Organisation ${ }^{36}$ und Fallkonstellation unterschiedlich zusammensetzt und in der Höhe variiert, die Unterstützung als selbstsüchtig erscheinen lässt. Die Rechtsprechung hat diese Frage verneint, sofern die Zahlungen des Sterbewilligen lediglich die ad-

31 Art. 128 StGB „Unterlassung der Nothilfe“ lautet wie folgt:

„Wer einem Menschen, den er verletzt hat, oder einem Menschen, der in unmittelbarer Lebensgefahr schwebt, nicht hilft, obwohl es ihm den Umständen nach zugemutet werden könnte, wer andere davon abhält, Nothilfe zu leisten, oder sie dabei behindert, wird mit Freiheitsstrafe bis zu drei Jahren oder Geldstrafe bestraft“"

32 Rettungssanitäter sind in der Schweiz zudem in der Regel keine Ärzte, sondern speziell ausgebildete Fachkräfte.

33 Vgl. dazu Reanimationsentscheidungen, medizinisch-ethische Richtlinien und Empfehlungen der SAMW vom 27. 11. 2008 (geändert am 01. 01. 2013), abrufbar unter: http://www.samw.ch/ de/Ethik/Richtlinien/Aktuell-gueltige-Richtlinien.html (Stand: 13. 01. 2016).

34 Fountoulakis/Köbrich, AJP 2013, 1440.

35 BGE 136 II 415, 420. Der (historische) Gesetzgeber hat nicht an den Fall einer Tätigkeit einer organisierten Suizidhilfe gedacht.

36 Vgl. z. B. Exit Deutsche Schweiz, Rahmen 45 CHF (Jahresmitgliedsbetrag) bis 3.500 CHF (zuzüglich allfälliger Zusatzkosten, wenn die Mitgliedschaft noch keine drei Jahre besteht), abrufbar unter: http://www.exit.ch/mitglied-werden (Stand: 13. 01. 2016), Dignitas, Rahmen ca. 7.000 CHF bis 10.500 CHF exklusive Mehrwertsteuer, abrufbar unter: http://www.dignitas.ch/index.php? option=com_content\&view=article\&id=22\&Itemid=62 (Stand: 13.01 .2016 ). 
ministrativen Kosten der Sterbehilfeorganisation decken ${ }^{37}$. Darüber hinaus besteht Unklarheit, wo die Grenzen zwischen einer zulässigen Finanzierung einer Organisation, welche eine Sterbebegleitung anbietet, und einer strafbaren selbstsüchtigen Gewinnabsicht liegen. Sollten die Mitgliederbeiträge an die Organisation neben den Aufwendungen und Spesen auch die angemessene Entlöhnung der Sterbebegleiter umfassen, könnte dies je nach Höhe und Regelmässigkeit in den strafbaren Bereich fallen ${ }^{38}$. Sollte von der Organisation mehr eingefordert werden, als für die Deckung von Aufwendungen und Spesen erforderlich ist, muss die Rechtsprechung im Einzelfall entscheiden, ob bereits von selbstsüchtigen Motiven gesprochen werden kann. Klare Richtlinien hierzu gibt es insoweit nicht $^{39}$.

Bestrebungen des nationalen Gesetzgebers, den Bereich der organisierten Suizidbeihilfe rechtlich zu regulieren, bestehen derzeit nicht. Aufgrund verschiedener Aufforderungen, minimale Sorgfalts- und Beratungspflichten für Suizidorganisation zu treffen, beauftragte der Bundesrat im Juli 2008 das Eidgenössische Justiz- und Polizeidepartement (EJPD) vertieft abzuklären, ob im Bereich der organisierten Sterbehilfe spezifische gesetzliche Regelungen erforderlich seien. Namentlich im Einsatz von Helium anstatt des verschreibungspflichtigen Natrium-Pentobarbitals ${ }^{40}$ durch Dignitas erblickte der Bundesrat eine Tendenz der organisierten Sterbehilfe, sich der ärztlichen Kontrolle zu entziehen sowie eine damit verbundene mögliche Missbrauchsgefahr. Ein umfassendes Aufsichtsgesetz fasste der Bundesrat allerdings nicht ins Auge, da dies zu einer Mitverantwortung des Staates führen und den Organisationen eine staatliche Berechtigung verleihen würde ${ }^{41}$. Im Rahmen der Abklärungen wurden zwei Varianten eines Gesetzesvorentwurfs ausgearbeitet und zur Vernehmlassung gestellt ${ }^{42}$. Eine Variante stellte die Suizidhilfe von Suizidhilfeorganisationen generell unter Strafe, eine andere formulierte Sorgfaltsanforderungen für Suizidhilfeorganisationen, deren Beachtung zur Straffreiheit führen sollte. Obgleich sich eine deutliche Mehrheit der Kantone, Parteien und interessierten Organisationen für eine ausdrückliche bundesrechtliche Regelung der organisierten Suizidhilfe ausgespro-

37 Schwarzenegger, in: BSK (Anm. 18), Art. 115 StGB Rdn. 14; BGer vom 17. 11. 2011, 1B_516/2011, E. 2.2.

38 Venetz, Suizidhilfeorganisationen im Strafrecht, 2008, S. $266 \mathrm{ff}$.

39 BGervom 17. 01. 2011, 1B_516/2011, E. 2.4.

40 Zur Rechtmäßigkeit der Verschreibungspflicht - auch mit Blick auf das Recht der persönlichen Freiheit, Art. 8 EMRK, Art. 10 BV, vgl. BGer Urt. 2A. 48/2006; 2A. 66/2006; BGE 133 I 58, 66 ff. 41 Abrufbar unter: https://www.bj.admin.ch/bj/de/home/aktuell/news/2008/ref_2008-07-020. html (Stand: 13. 01. 2016).

42 https://www.bj.admin.ch/dam/data/bj/gesellschaft/gesetzgebung/archiv/sterbehilfe/entwd.pdf (Stand: 13. 01. 2016). 
chen hatten, konnte kein Konsens über das „Wie“ erzielt werden. Daher gelangte der Bundesrat am 29. 06. 2011 zum Schluss, auf eine ausdrückliche Regelung der organisierten Suizidhilfe $\mathrm{zu}$ verzichten ${ }^{43}$. Die Verpflichtungen, welche durch Erlass zusätzlicher Sorgfaltspflichten hätten begründet werden sollen, seien in den geltenden gesetzlichen Regelungen bereits genügend enthalten. Hingegen würde eine Gesetzesänderung die Suizidhilfeorganisationen staatlich legitimieren. Dies könne einen Anreiz schaffen, die Dienste zusätzlich in Anspruch zu nehmen. Eine solche Legitimierung durch den Staat könne die Unantastbarkeit des menschlichen Lebens relativieren. Ferner hatte der Bund bei seiner Entscheidung Rücksicht auf die Ärztekreise genommen, welche sich gegen eine ärztlich vorgenommene Suizidhilfe ausgesprochen hatten. Der Bundesrat sprach sich zugleich dafür aus, die Förderung im Bereich der Suizidprävention sowie der Palliative Care zur Betreuung und Behandlung von Menschen mit unheilbaren, lebensbedrohlichen und/oder chronisch fortschreitenden Krankheiten weiter $\mathrm{zu}$ intensivieren ${ }^{44}$.

Die Diskussion um die Rahmenbedingungen der Sterbehilfe ist damit allerdings nicht beendet. 2013 hatte der Bundesrat in der Antwort auf eine Anfrage von Nationalrat Gross ${ }^{45}$, die sich unter anderem auf den Wirkungskreis des Nationalen Forschungsprogramms „Lebensende“ (NFP 67) ${ }^{46}$ bezog, festgehalten, „[...] Dass [...] die heutige Praxis der Sterbehilfe untersucht und gegebenenfalls Empfehlungen und Änderungsvorschläge ausgearbeitet werden, wird gemäss Entscheiden des Bundesrates vom NFP 67 erwartet“"47. 2015 hat der Bundesrat sich auf eine Interpellation hin dahingehend eingelassen, dass er die Vorschrift des Art. 115 StGB für ausreichend erachtet, um das Vorliegen von selbstsüchtigen und insbesondere finanziellen Motiven bei Sterbehilfeorganisationen $\mathrm{zu}$ kontrollieren. Dies setzte jedoch eine konsequente Abklärung durch die Strafverfolgungsbehörden voraus. Die Einführung einer speziellen Finanzaufsicht über Sterbehilfeorganisationen sei weder notwendig noch zielführend ${ }^{48}$.

Auch die Kantone sind im Bereich von Regelungen der Sterbehilfe eher zurückhaltend. Davon ausgenommen ist der Kanton Waadt, in welchem am

43 Vgl.https://www.bj.admin.ch/bj/de/home/aktuell/news/2011/ref_2011-06-29.html (Stand: 13.01.2016).

44 Vgl. http://www.ejpd.admin.ch/ejpd/de/home/aktuell/news/2011/2011-06-29.html (Stand: 13. 01. 2016).

45 Gross, 13.1006 - Anfrage, abrufbar unter: http://www.parlament.ch/d/suche/seiten/ge schaefte.aspx?gesch_id=20131006 (Stand:13. 01. 2016).

46 Näher dazu: http://www.nfp67.ch/de/Seiten/Home.aspx (Stand: 13. 01. 2016).

47 Vgl. http://www.parlament.ch/d/suche/seiten/geschaefte.aspx?gesch_id=20131006 (Stand: 13. 01. 2016).

48 Glanzmann-Hunkeler, Interpellation, 15.3947 mit Antwort des Bundesrates vom 25. 11. 2015. 
17. 06. 2012 mit 61,6\% der Wähler und Wählerinnen ein Gesetz akzeptiert wurde, das Sterbehilfe unter bestimmten Bedingungen in öffentlich finanzierten Pflegeeinrichtungen und Hospitälern zulässt ${ }^{49}$. Die von Exit Romandie Suisse 2009 lancierte Initiative „Assistance au suicide en EMS (établissements médico-sociaux)“, die keine einengenden Bedingungen enthielt, wurde hingegen mit 59,1\% der Stimmen zurückgewiesen.

\section{c) Fremdtötung}

\section{aa) Tötung durch aktives Tun}

Kommt der handelnden Person Tatherrschaft und damit Tatmacht über die Tötung eines anderen zu, liegt eine Fremdtötung vor, wenn der Entschluss erfolgreich umgesetzt wird. Insoweit gelten die Tötungsdelikte nach Art. 111 StGB, Tötung, Art. 112 StGB, Mord, Art. 113 StGB, Totschlag, Art. 114 StGB, Tötung auf Verlangen, sowie die fahrlässige Tötung, Art. 117 StGB. Ausschlaggebend für die Unterscheidung von Fremdtötung und Suizidhilfe sind also die Tatmacht und die Einflussnahme auf das Geschehen sowie die Art der Beweggründe des Täters. Hat die getötete Person ihre Tötung in ernsthafter und eindringlicher Weise verlangt und wird die Tötungshandlung aus achtenswerten Beweggründen, namentlich aus Mitleid, durch eine Drittperson vollzogen, liegt ein Fall der mit bis zu drei Jahren Freiheitsstrafe oder Geldstrafe bedrohten, privilegierten Tötung nach Art. 114 StGB vor. Das ernsthafte und eindringliche Verlangen des Getöteten sowie die achtenswerten Beweggründe beeinflussen ${ }^{50}$ das Tötungsunrecht und entfalten Sperrwirkung gegenüber den anderen Tötungsdelikten ${ }^{51}$. Die Schwelle hierfür ist freilich hoch: Das Bitten oder Verlangen des Sterbewilligen muss intensiv, d.h. in der Regel wiederholt, sein und auf einer ernsthaften, eigenverantwortlichen Entscheidung des Sterbewilligen gründen sowie von ihm ausgehen $^{52}$. Führt der Täter die Tötungshandlung zwar nicht auf Verlangen, aber doch im mutmaßlichen Willen des Opfers durch, gelten die Art. 111-113 StGB.

49 Die Person, die Sterbehilfe verlangt, leidet an einer unheilbaren schweren Krankheit und ist urteilsfähig. Zudem müssen palliative Maßnahmen mit dem Patienten besprochen worden sein und weder das Pflegepersonal noch der verantwortliche Arzt dürfen bei der Sterbehilfe beteiligt sein, abrufbar unter: http://www.vd.ch/themes/sante/professionnels/assistance-au-suicide (Stand: 13. 01. 2016).

50 Schwarzenegger, in: BSK (Anm. 18), Art. 114 Rdn. 2.

51 Schwarzenegger, in: BSK (Anm. 18), Art. 114 Rdn. 17.

52 Donatsch, Strafrecht III (Anm. 21), § 1, 5. 


\section{bb) Indirekte aktive Sterbehilfe}

Im Bereich der Fremdtötung ist auch in der Schweiz die Fallgruppe der indirekten aktiven Sterbehilfe anerkannt. Obgleich sie bei rein technischer Anwendung der allgemeinen Regeln nach Art. $111 \mathrm{ff}$. StGB strafbar wäre, wird sie von der Rechtspraxis aufgrund normativer Erwägungen unter restriktiven Bedingungen aus dem Bereich des Strafbaren ausgeklammert. Als Motiv des ärztlichen Handelns ist vorausgesetzt, dass in der Endphase des Lebens der sich ankündigende Sterbeprozess erleichtert wird, z. B. um durch die Gabe von Medikamenten die Vernichtungsschmerzen zu dämpfen, auch wenn die Lebensverkürzung als mögliche oder sichere Nebenfolge der Medikamentengabe in Kauf genommen wird ${ }^{53}$. Rechtsgrund der Straflosigkeit ist nicht etwa ein Gewohnheitsrecht oder gar eine allgemeine Berufspflicht des Arztes ${ }^{54}$, sondern die normative Wertung, der zufolge die Schutzbereiche der Delikte zum Schutz von Leib und Leben dieses Handeln nicht erfassen ${ }^{55}$.

\section{cc) Unterlassen lebensverlängernder ärztlicher Behandlung}

Die Schweiz unterscheidet ${ }^{56}$ nach wie vor zwischen aktiver und unter engen Voraussetzungen als zulässig erachteter ${ }^{57}$ passiver Sterbehilfe. Letztere ist durch die Nichtaufnahme oder Einstellung einer lebenserhaltenden ärztlichen Behandlung gekennzeichnet, wenn dadurch dem Sterbeprozess freier Lauf gelassen wird. $\mathrm{Zu}$ den lebenserhaltenden Maßnahmen gehören insbesondere die künstliche Wasser- und Nahrungszufuhr, die künstliche Beatmung, die kardiopulmo-

53 Riklin, in: Holderegger (Hrsg.), Das medizinisch assistierte Sterben, Zur Sterbehilfe aus medizinischer, ethischer, juristischer und theologischer Sicht, 1999, S. 332, $337 \mathrm{ff}$. m.w.N.; Stratenwerth/Jenny/Bommer, Bes. Teil (Anm. 25), §1 Rdn. 8; Trechsel/Geth, in: Trechsel/Pieth (Hrsg.), StGB Praxiskommentar, 2. Aufl. 2013, Vor Art. 111 Rdn. 7 m.w. N.; BGHSt. 42, 301 ff. Es kann aber durchaus auch sein, dass durch eine gute Schmerzkontrolle das Leben des Patienten verlängert wird, vgl. Borasio, selbst bestimmt sterben, 2014, S. $47 \mathrm{ff}$.; ders., Über das Sterben. Was wir wissen. Was wir tun können. Wie wir uns darauf einstellen, 2011, S. $68 \mathrm{ff}$. 54 So z. B. Arbeitsgruppe „Sterbehilfe“, Bericht vom März 1999, S. 43 f., abrufbar unter: https:// www.bj.admin.ch/dam/data/bj/gesellschaft/gesetzgebung/archiv/sterbehilfe/b-bericht-d.pdf (Stand: 13. 01. 2016).

55 Tag, in: Kuhn/Poledna (Anm. 17), S. 737; zu weiteren Begründungsansätzen vgl. Schwarzenegger, in: BSK (Anm. 18), Vor Art. 111 Rdn. 62.

56 Anders die Beurteilung in Deutschland vgl. BGHSt. 454, 09 = NJW 2010, $2963 \mathrm{ff}$.

57 Bericht Sterbehilfe und Palliativmedizin - Handlungsbedarf für den Bund? 24. 04. 2006, Ziff. 4.2.1., abrufbar unter: https://www.bj.admin.ch/dam/data/bj/gesellschaft/gesetzgebung/archiv/ sterbehilfe/20060531_ber-sterbehilfe-d.pdf (Stand: 13. 01. 2016). 
nale Reanimation und - je nach Situation - die Sauerstoffzufuhr, Medikation, Transfusion, Dialyse sowie operative Eingriffe ${ }^{58}$. Die passive Sterbehilfe wird interpretiert als ein Unterlassen, hier des Arztes oder Pflegepersonals, das den Eintritt des Todes zur Folge hat. Die Bestimmung von Art. 11 StGB, Begehen durch Unterlassen, ist auf diese Konstellationen anwendbar, wobei sich die Garantenstellung des behandelnden Arztes in der Regel aus der Behandlungsübernahme ergibt. Die hiermit verbundenen Garantenpflichten, so z.B. die Pflicht, einzugreifen und das Leben sowie die Gesundheit des Patienten zu erhalten, werden zum einen durch den (Gesundheits-)Zustand ${ }^{59}$ und zum anderen durch die autonomen Entscheidungen des Patienten beeinflusst. Hat er frei verantwortlich, d.h. in urteilsfähigem Zustand, die aktuell gültige Entscheidung gegen lebensverlängernde Maßnahmen getroffen, so ist der Arzt daran gebunden. Der Entschluss für einen Behandlungsabbruch ist von der Behandlungsseite grundsätzlich zu respektieren, selbst wenn dadurch das Leben des Patienten verkürzt wird. In dieser Konstellation wird aber nicht die Garantenstellung ${ }^{60}$ des Arztes beendet. Denn der Patient ist in der Regel auch in der Phase des Sterbens auf den Arzt angewiesen, um in Würde und menschengerecht sterben zu können. Dazu gehören auch die Anwendung von palliativen Maßnahmen zur Schmerzkontrolle sowie menschliche Zuwendung ${ }^{61}$. Tritt der Tod infolge des Verzichts auf lebensverlängernde Maßnahmen ein, ist dieses Ereignis dem Arzt nicht als Verletzung einer ärztlichen Garantenpflicht zuzurechnen ${ }^{62}$. Es fällt vielmehr als freiverantwortliche Entscheidung in den Verantwortungsbereich der sterbewilligen Person.

Große Fragen stellen sich, wenn der Patient zum Zeitpunkt, zu dem lebenserhaltende Maßnahmen notwendig sind, endgültig nicht mehr urteils- und damit entscheidungsfähig ist. Hier gibt das auf den 01. 01. 2013 in Kraft getretene Schweizerische Erwachsenenschutzrecht Hilfestellungen. Das vom Gedanken der Autonomie des Einzelnen getragene Regelwerk enthält u. a. explizite Regelungen

58 SAMW-Richtlinien zur Betreuung von Patientinnen und Patienten am Lebensende vom 25. 11. 2004 (aktualisiert 2012), Kommentar 3.2, abrufbar unter: http://www.samw.ch/de/Ethik/ Richtlinien/Aktuell-gueltige-Richtlinien.html (Stand: 13. 01. 2016).

59 Vgl. Art. 17 Abs. 1 Standesordnung FMH.

60 Anders der Bericht Sterbehilfe und Palliativmedizin - Handlungsbedarf für den Bund? 24. 04. 2006, Ziff. 4.2.1, abrufbar unter: https://www.bj.admin.ch/dam/data/bj/gesellschaft/ gesetzgebung/archiv/sterbehilfe/20060531_ber-sterbehilfe-d.pdf(Stand: 13. 01. 2016).

$61 \mathrm{Vgl}$. dazu ebenfalls die Richtlinien zur Betreuung von Patientinnen und Patienten am Lebensende vom 25. 11. 2004 (aktualisiert 2012), abrufbar unter: http://www.samw.ch/de/Ethik/Richtlin ien/Aktuell-gueltige-Richtlinien.html (Stand: 13. 01. 2016).

62 Die Bedeutung der Patientenautonomie unterstreicht Art. 17 Abs. 1Standesordnung FMH. 
zur Patientenverfügung ${ }^{63}$ und zur sonstigen Vertretung der urteilsunfähigen Person. Die Patientenverfügung betrifft die Frage, welche medizinischen Maßnahmen für den Fall der Urteilsunfähigkeit angewendet oder unterlassen werden sollen und hat - soweit die gesetzlichen Vorgaben, vgl. Art. $370 \mathrm{ff}$. ZGB, eingehalten sind - Verbindlichkeit. Dies gilt nicht, wenn die Anweisungen gegen gesetzliche Vorschriften verstossen (bspw. direkte aktive Sterbehilfe oder sittenwidrige Anordnungen einfordern), der freie Wille des Verfassers begründet angezweifelt werden kann oder dem mutmaßlichen Willen der Person nicht mehr entspricht. Abweichungen von einer Patientenverfügung müssen in der Patientendokumentation festgehalten werden, Art. 372 Abs. 3 ZGB. Zudem können nahestehende Personen des Verfügenden bei der Erwachsenenschutzbehörde geltend machen, dass die Interessen der urteilsunfähigen Person nicht mehr gewahrt werden, Art. 373 Abs. 1 Ziff. 1 ZGB.

Für den Fall, dass sich der Patient z. B. im irreversiblen Wachkoma befindet und der eigentliche, irreversible Sterbeprozess noch nicht begonnen hat, darf der Arzt daher nicht ohne weitere Abklärungen von sich aus die Ernährung auf eine reine Flüssigkeitszufuhr umstellen. Denn unabhängig vom Bewusstseinszustand des Patienten ist das Leben bis zum Eintritt des Gesamthirntodes geschützt. Wie vorzugehen ist und ob einem dauernd bewusstlosen oder aus anderen Gründen irreversibel urteilsunfähigen Patienten passive Sterbehilfe geleistet werden darf, bestimmt sich zunächst nach den allgemeinen Grundsätzen ärztlicher Behandlung. Bei urteilsunfähigen Personen haben, unter Vorbehalt der Beachtung des in einer allfälligen Patientenverfügung geäußerten Willens, deren gesetzliche resp. die von der betreffenden Person bestimmten Vertreter ${ }^{64}$ (bei Minderjährigen grundsätzlich ${ }^{65}$ die Eltern bzw. der Vormund, ansonsten vgl. Art. 378 ZGB) über die Anwendung bzw. Weiterführung lebensverlängernder Maßnahmen zu befinden. Sie müssen sich hierbei an dem mutmaßlichen Willen und den Interessen der urteilsunfähigen Person ausrichten, Art. 378 Abs. 3 ZGB. In dringlichen Fällen ergreift der Arzt die medizinische Maßnahme nach dem mutmaßlichen Willen und den Interessen des urteilsunfähigen Patienten, vgl. Art. 379 ZGB. Ist eine entsprechende Abklärung nicht rechtzeitig möglich, müssen die zur Lebensrettung oder zur Abwehr schwerer Folgeschädigungen unaufschiebbaren Maßnahmen sofort eingeleitet werden, wenn ein Hinausschieben des unvermeidbaren

63 Zum Ganzen: Häfeli, AJP 2014, 1593. Allerdings gibt es kein Recht des Einzelnen, dass Dritte oder der Staat zu einem positiven Tun oder einer positiven Pflicht verpflichten kann, Hilfe beim Sterben zu leisten, BGE 133 I 58, 68.

64 Vorbehalten ist zudem ein Eingreifen der Kindes- resp. Erwachsenenschutzbehörde.

65 Auch urteilsfähige Minderjährige können eine Patientenverfügung i.S.v. Art. 370 ff. ZGB errichten. 
Todes für die sterbende Person nicht lediglich eine unzumutbare Verlängerung des Leidens bedeuten würde ${ }^{66}$. Sobald Kenntnis von einer gemäß Art. 372 Abs. 2 ZGB zu entsprechenden Patientenverfügung besteht, ist diese für das weitere Vorgehen maßgebend. Bestehen begründete Zweifel, dass die Patientenverfügung dem mutmaßlichen Willen des Patienten entspricht, sind, wenn immer möglich, seine nächsten Bezugspersonen zu befragen, um Anhaltspunkte für die zutreffende Beurteilung jenes Willens $\mathrm{zu}$ erhalten ${ }^{67}$. Liegt weder eine (i.S.v. Art. 372 Abs. 2 ZGB zu beachtende) Patientenverfügung, der eine Willensäußerung zur fraglichen Behandlung entnommen werden kann, noch ein dringlicher Fall i.S.v. Art. 379 ZGB vor, obliegt der Entscheid über die medizinischen Maßnahmen grundsätzlich dem gesetzlichen Vertreter. Auch dieser hat, vorausgesetzt dass in einer Patientenverfügung Weisungen fehlen, nach dem mutmaßlichen Willen und den Interessen der urteilsunfähigen Person ${ }^{68} \mathrm{zu}$ entscheiden, Art. 378 Abs. 3 ZGB. Es geht dabei um die Einschätzung darüber, wie sich der Patient im jetzigen Zeitpunkt entscheiden würde, wenn er urteilsfähig und über seinen Zustand und die Prognose seines Leidens vollumfänglich aufgeklärt wäre. $\mathrm{Zu}$ berücksichtigen sind u.a. die früher gemachten Äußerungen, religiöse und sonstige Wertehaltungen sowie biographische Hinweise.

\section{Ausblick}

Die vorstehenden Bemerkungen zeigen, dass die Sterbehilfe und die Hilfe zum Suizid in der Schweiz nicht nur ethisch relevante, sondern gerade auch zentrale (straf-)rechtliche Themen sind. Die aufgeworfenen Fragen gewinnen zudem eine neue Dimension, wenn die Hilfe zum Sterben nicht im Einzelfall - sei es durch Angehörige, Freunde oder auch einen Arzt - sondern professionell organisiert angeboten und durchgeführt wird.

Auch wenn der Bundesrat beschlossen hat, die Reform von Art. 115 StGB nicht weiter zu verfolgen, ist die Sterbehilfedebatte damit nicht beendet. Zwar hat er ein Zeichen dahingehend gesetzt, die Selbstbestimmung des Einzelnen in weitem Masse zu respektieren, gerade auch wenn er sich für den Tod entscheidet. Aber dennoch kann in diesen zentralen Fragen des Lebens und der Grundrechte, die nicht nur individuelle Abwehrrechte gegen den Staat sind, sondern ihn auf institutioneller Ebene auch verpflichten einer Aushöhlung entgegenzuwirken,

66 Vgl. Art. 17 Abs. 1 Standesordnung FMH.

67 Vgl. dazu Hausheer/Geiser/Aebi-Müller, Das Familienrecht des Schweizerischen Zivilgesetzbuches, 5. Aufl. 2014, § 20 Rdn. 20.53.

68 Bei Minderjährigen ist das Kindeswohl entscheidend. 
schwerlich darauf verzichtet werden, bindende Sorgfaltskriterien $\mathrm{zu}$ definieren. So muss bereits aus strafrechtlicher Sicht überprüft werden, ob die sterbewillige Person ihren Willen frei gefasst und geäußert hat, er wohlerwogen ist und auf Dauer besteht. Zudem ist zu klären, wie bei urteilsunfähigen Personen mit und ohne gültige Patientenverfügung zu verfahren ist. Ein weiterer Fragenkreis betrifft die Beteiligung von Ärzten. Ist es notwendig, wünschenswert oder unerwünscht, aber $\mathrm{zu}$ tolerieren ${ }^{69}$, dass sie den Suizid begleiten? Welche Abklärungen sind dann im Vorfeld zu treffen? Sollte es Voraussetzung für die Unterstützung durch organisierte Sterbehelfer sein, dass die Person terminal krank ist, an einer genügend schweren chronischen Krankheit leidet oder geht die persönliche Freiheit so weit, dass der unterstützte Freitod gesunden, aber lebensmüden bzw. psychisch kranken Personen zur Verfügung stehen soll? Und soll bzw. darf der Suizid in einem Spital oder einer sonstigen öffentlich-rechtlichen Pflegeeinrichtung durchgeführt werden? Der Bundesrat hat in seiner Stellungnahme vom Juni 2011 betont, dass auf die Suizidprävention großes Gewicht gelegt werden soll.

Die Fragen, die Antworten suchen, sind zahlreich. Die Verantwortung, die damit verbunden ist, ist groß. Die jüngste Geschichte zu einer allfälligen Reform von Art. 115 StGB zeigt, dass die Lösungen sich nicht von heute auf morgen finden lassen. Eine Hoffnung ist, dass das vom Schweizerischen Nationalfonds initiierte „Nationale Forschungsprogramm Lebensende, NFP 67“ auch in diesem Bereich neue Erkenntnisse bringt. Es hat zum Ziel, neues Wissen über die letzte Lebensphase von Menschen jeden Alters zu gewinnen, die aller Voraussicht nach, nur noch kurze Zeit zu leben haben. Prägend ist hierbei die Erkenntnis, dass Entscheidungen in den letzten Lebensmonaten nicht nur medizinischer Art sind. Sie sind auch geprägt von gesellschaftlichen Normen, rechtlichen Regelungen, Werthaltungen, religiösen Bekenntnissen und persönlichen Wünschen. Im NFP 67 wird das Lebensende daher aus unterschiedlichen Perspektiven erforscht, wobei es um Sterbeverläufe und Versorgung, Entscheidungen, Motive und Haltungen, Regelungen und Handlungsvorschläge geht. Zudem hat sich die Nationale Ethikkommission im Bereich Humanmedizin die „Kultur des Sterbens“ als ein Schwerpunktthema vorgenommen, um damit die ethischen Fragen der facettenreichen Möglichkeiten und Schwierigkeiten eines möglichst guten, menschenwürdigen Sterbens aufzuarbeiten. ${ }^{70}$ Last but not least besteht in der Schweiz ein großes Interesse, die Palliative Care auszubauen und in den Versorgungsstrukturen besser zu verankern.

69 Dazu die medizin-ethischen Richtlinien der SAMW zur Betreuung von Patientinnen und Patienten am Lebensende vom 25. 11. 2004 (Anm. 61).

70 Vgl. http://www.nek-cne.ch/de/die-kommission/arbeitsgruppen/index.html (Stand: 13. 01. 2016). 
Das alles zusammengefasst zeigt, dass die Diskussion um das Lebensende und den (assistierten) Suizid gerade auch in der Schweiz viele Dimensionen hat. Es bleibt zu wünschen, dass im Zentrum aller Bemühungen und Diskussionen der Mensch steht und es möglich ist, ihm in der sehr vulnerablen Situation des Alters und der Krankheit angemessene Hilfe und Fürsorge, namentlich durch gut ausgebaute Palliativversorgung und Hospize zu bieten, um in Würde zu leben und auch sterben zu können.

Danksagung: Die Verf. dankt Frau MLaw Isabel Baur für die Unterstützung bei der Erstellung dieses Beitrages. 